\title{
MÚSICA, NECESSIDADES EDUCATIVAS ESPECIAIS E INCLUSÃO ${ }^{1}$
}

\author{
Music, special educational needs and inclusion
}

ROCHA, Alberto², \& CARDOSO, Mário ${ }^{3}$

\begin{abstract}
Resumo
Neste trabalho apresentam-se os resultados de um estudo em torno da importância da utilização das Tecnologias de Informação e Comunicação (TIC), no processo de ensino e aprendizagem de uma aluna portadora de Trissomia 21 (T21), que reforça conceitos musicais com recurso ao Soundbeam. Orientado pelos princípios da Educação Inclusiva e pelo pressuposto de que as TIC têm impacto nos processos de Ensino e de Aprendizagem, particularmente naqueles destinados a alunos portadores de Necessidades Educativas Especiais (NEE) e especificamente de T21. Realizou-se uma revisão bibliográfica visando constituir o enquadramento teórico da problemática do estudo, pretendendo compreender e colmatar uma dificuldade de marcação do ritmo, recorrendo a algumas estratégias como meio para atingir objetivos e resultados positivos, no processo de ensino aprendizagem. Os resultados apurados da aplicação permitiram constatar o elevado grau de motivação e interesse revelados pela jovem no uso das TIC, bem como a sua capacidade em utilizar equipamentos de uso generalizado e não especificamente adaptados.
\end{abstract}

\begin{abstract}
This work presents the results of a study about the importance of the use of Information and Communication Technologies (ICT) in the teaching and learning process of a female student with Trisomy 21 (T21), which reinforces musical concepts using Soundbeam. Guided by the principles of Inclusive Education and the assumption that ICT have an impact on the processes of Teaching and Learning, particularly those destined to students with Special Educational Needs (SEN) and specifically T21. A bibliographical review was carried out aiming at constituting the theoretical framework of the problem of the study, aiming at understanding and filling a difficulty of marking the rhythm, resorting to some strategies as a means to achieve goals and positive results in the process of teaching learning. The verified results of the application allowed to verify the high degree of motivation and interest revealed by the young person in the use of the TIC, as well as their capacity to use equipment of generalized use and not specifically adapted.
\end{abstract}

Palavras-chave: Música; Inclusão; TIC; NNE; T21.

Key-words: Musica; Inclusion; ICT; SEN; T21.

Data de submissão: março de 2018 | Data de publicação: março de 2019.

\footnotetext{
${ }^{1}$ Investigação / publicação decorrente da comunicação apresentada no II Simpósio Internacional de Investigação em Arte "Arte \& Inclusão": 19 e 20 de abril de 2017 (Vila Real, Amarante e Lamego).

2 ALBERTO ROCHA - Instituto Superior de Ciências Educativas do Douro, PORTUGAL. E-mail: ar631795769@gmail.com

${ }^{3}$ MÁRIO CARDOSO - Escola Superior de Educação de Bragança do Instituto Politécnico de Bragança, PORTUGAL. E-mail: cardoso@ipb.pt
} 


\section{INTRODUÇÃO}

O início do século XXI ficará marcado por um conjunto de iniciativas transformadoras em educação, configurando um novo paradigma no qual a Inclusão se tornou num modelo educativo pelo qual as escolas se devem orientar. Procurando dar resposta às mudanças da sociedade atual, a Escola vê-se em situação de procurar soluções que se ajustem às necessidades e caraterísticas de uma população diversificada, resultado da democratização e massificação do sistema de ensino. Nas nossas escolas parece estarem criadas as condições propícias a uma Inclusão genuína, através de e com as TIC, nas aprendizagens dos nossos alunos.

Com a publicação do Decreto-Lei 3/2008, de 7 de janeiro, cujos princípios estão enraizados nos valores democráticos e inclusivos consolidados no século XX e enunciados na Declaração de Salamanca, 1994, traçando um novo rumo face aos modelos organizacionais e às políticas educativas. Paralelamente, surgiu nas escolas, uma revolução tecnológica com o objetivo de preparar "as novas gerações para os desafios da sociedade do conhecimento" (O PTE - Missão e Objetivos, 2009), através do desenvolvimento de competências em TIC e da sua integração nos processos de ensino/aprendizagem, dos quais emerge a necessidade do apetrechamento informático das escolas, do desenvolvimento de conteúdos, da formação dos professores em TIC e do fomento do uso das TIC pelos cidadãos com necessidades especiais.

Partindo destas questões, procurámos contribuir para a organização e aprofundamento do conhecimento constituído em torno das TIC e da deficiência, visto que os alunos portadores de deficiência, serão beneficiários prioritários destas medidas, que esbatem diferenças e promovem a "partilha sem barreiras" (O PTE -Missão e Objetivos, 2009) e de entre estes, demos destaque aos que são portadores de T21, pela pertinência de colaborar na sistematização da informação sobre esta tipologia de deficiência, tantas vezes mal compreendida em contexto educativo. $\mathrm{O}$ estudo procurou conhecer em que medida as TIC constituem uma ferramenta potencializadora da aprendizagem para alunos portadores de $\mathrm{T} 21$. 


\section{O SÍNDROME DE DOWN /TRISSOMIA 21}

Em 1886, John Lang Down, médico britânico, publicou um artigo em que descrevia algumas características morfológicas da síndrome que hoje se denomina de "Síndrome de Down" (SD) ou Trissomia 21 (T21). Isto permitiu distinguir estas crianças de outras que também apresentavam deficiência mental. A T21 é, assim, uma das categorias mais comuns de deficiência mental clinicamente classificada. É um problema de origem genética que se caracteriza pela presença de três cópias do cromossoma 21 nas células (Vinagreiro \& Peixoto, 2000).

A educação de uma criança com T21 deve ser orientada pelo desenvolvimento das suas faculdades cognitivas e sociais específicas até ao mais alto grau que lhes for possível, segundo Lopez (1983), citado por Sampedro, Blasco e Hernandez , (1997), assim como pelo princípio da normalização, segundo o qual a criança deve ter acesso aos serviços gerais da comunidade, para que possa, deste modo integrar-se nela (Real _decreto de La Ordenacion de la Educacion Especial, 1985, (Sampedro, Blasco, \& Hernandez, 1997).

Como a T21 é uma deficiência diagnosticada precocemente, a criança poderá beneficiar de um bom programa de intervenção precoce, dirigido por uma equipa multidisciplinar em conjunto com a sua família. Segundo Sampedro, Blasco e Hernandez (1997, p. 236) “(...) o programa deverá contemplar todas as áreas de desenvolvimento: psicomotricidade fina e grossa, linguagem e comunicação, socialização e autonomia pessoal, desenvolvimento afetivo e cognitivo". O desenvolvimento destas áreas fornecerá as bases essenciais para a aquisição de desempenhos mais complexos ao nível das aprendizagens académicas, nomeadamente, aprendizagem da leitura/escrita e matemática, de forma a criar futuros cidadãos ativos e autónomos na sociedade em que se encontram inseridos.

\section{CONCEITO E CARATERÍSTICAS}

T21, em que a mesma apresenta um cromossoma extra em cada célula, definido como cromossoma 21 ou também denominado T21 (Ataíde, 2008, p. 26), pelo que, uma pessoa “normal", possui 46 cromossomas, na qual estão divididos em 23 pares, enquanto que um portador de $\mathrm{SD}$, o par de numero 21 possui um cromossoma a mais, dando origem a 47 cromossomas. 
De acordo com Lefévre, citado por Barroso (2010, p. 17), a "pessoa com T21 tem capacidade para realizar várias tarefas relacionadas com o espaço, o tempo, com a organização gráfica, o raciocínio lógico e uma sensibilidade corporal, mas para que seja possível é necessária uma intervenção adequada como meio de estímulo e retenção da aprendizagem". Segundo Leme, citado por Anunciação, Costa e Denori (2015), ao depararmo-nos com dificuldades, "teremos de pensar em soluções, acreditando que a educação é transformadora de uma forma realista".

É relevante implementar programas de estimulação com atividades que favoreçam o desenvolvimento motor, devendo ser lúdicas e atrativas para a criança. A T21, também designada em termos morfológicos fenótipo, "define-se como uma alteração da organização genética e cromossómica do par 21, pela presença total ou parcial de um cromossoma (autossoma) extra nas células do organismo ou por alterações de um dos cromossomas do par 21, por permuta de partes com outro cromossoma de outro par de cromossomas" (Morato, 1995, p. 23). A alteração verificada resulta de um processo irregular da divisão celular, podendo ocorrer de forma acidental, circunstancial ou por uma predisposição hereditária (materna ou paterna). A T21 é, assim uma designação genética que identifica as pessoas que nascem com sinais morfológicos específicos variantes (fenótipo típico), correspondendo a uma organização cromossómica determinada, ainda que diferentes segundo três modalidades ou tipos:

Trissomia 21 regular - é a modalidade mais frequente (95\%), sendo explicada pela presença de um cromossoma extra no par 21, em todas as células, devido à não disjunção dos cromossomas na divisão celular.

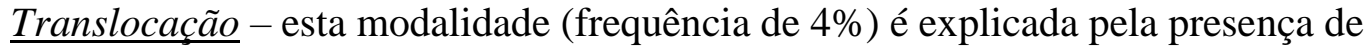
uma parte de um dos cromossomas do par 21, trocada com outra parte de outro cromossoma de outro par de cromossomas como do par 13, 14, 15, 22, sendo o 14 o mais frequente.

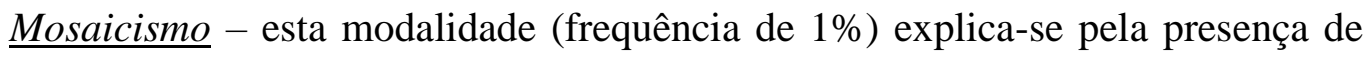
parte extra do cromossoma 21 somente em alguma proporção das suas células. 


\section{TIC E EDUCAÇÃO ESPECIAL}

A influência das TIC na educação traduz-se em novos cenários que geram novas oportunidades para os todos alunos, quer tenham NEE ou não. As TIC não surgem como um único fator de inovação escolar, são, provavelmente, o instrumento que leva às transformações em educação e fazem com que esta se adapte a uma realidade social promotora de igualdade, acessibilidade e respeito pela diversidade. As TIC são, então, uma das áreas chave dos serviços de apoio à inclusão, pelo seu enorme potencial para melhorar a qualidade da educação dos alunos com NEE. Segundo Amante (2007, p. 52) é "difícil ignorar o contributo destes novos media no enriquecimento da aprendizagem" e, no entanto, este contributo depende de um uso prolífico da tecnologia, em que "a resposta possível não está na tecnologia, mas sim nas pessoas e nas instituições” perante o desafio de perspetivar as TIC como motores de mudança "para um modelo de funcionamento baseado na construção partilhada do conhecimento, aberto à diversidade" (Silva, 2001, p. 23). A educação especial (EE) desenvolveu-se em torno da igualdade de oportunidades, em que todos os indivíduos, independentemente das suas diferenças, deverão ter acesso a uma educação com qualidade capaz de responder a todas as suas necessidades.

Assim sendo, esta educação deve desenvolver-se de forma especial, numa tentativa de atender às diferenças individuais de cada criança, através de uma adaptação do sistema educativo (Correia, 1997). Ao longo do processo de aprendizagem e desenvolvimento das crianças, em especial das que apresentam défice cognitivo é fundamental identificar problemáticas e características individuais, para que seja possível estabelecer intervenções pedagógicas adequadas e assertivas. As competências práticas e funcionais devem ser realçadas para que possam ser atingidas em situações reais do quotidiano, visando uma maior independência pessoal e social, um desenvolvimento dos aspetos sócio cognitivos e da autonomia (Ribeiro, 2008).

Melhorar a qualidade da educação passa também por saber tirar partido da tecnologia, pô-la ao serviço de um projeto educativo renovado em que, para além do que se aprende, se aprende a aprender. Para que esse projeto se renove, cabe ao sistema educativo e aos professores, agilizarem os meios possíveis para utilizar cada vez mais as TIC como ferramentas no processo de ensino e aprendizagem e como tecnologias de apoio aos alunos com NEE (Amante, 2007). 
O potencial das TIC, quer no que se refere à natureza dos programas utilizados, quer às possibilidades de acesso à informação e comunicação disponíveis, revelam um contributo no enriquecimento dos contextos de aprendizagem.

\subsection{As TIC e as NEE}

O sentido básico da EE é considerar a personalidade como um todo que envolve perceção, cognição, emoção, motivação e socialização. Não devemos centrar-nos apenas na incapacidade de determinados indivíduos considerados como NEE, mas sim, minimizar essas capacidades para que possam fazer um percurso escolar e social o menos limitado possível e num meio menos restrito possível, como preconiza a Declaração de Salamanca (1994).

Neste cenário de mudança em que, segundo Lloyd, Moni e Jobling (2006), as crianças e jovens crescem mergulhadas em tecnologia que altera o ambiente no qual se desenrolam as aprendizagens, as TIC representam um elemento decisivo na normalização das condições de vida dos alunos com NEE e, podem mesmo ser a única possibilidade que estes têm de aceder ao currículo que, de outro modo, lhes estaria barrado.

Considerando as TIC como motores de mudança, podemos identificar vantagens para os alunos com NEE, como propõem Pérez e Montesinos (2007), que se prendem com a superação de limitações, com o benefício da autonomia e da formação individualizada, favorecendo a diminuição do sentido de fracasso, enquadrando-se num modelo multissensorial propício à aproximação dos sujeitos ao mundo.

O relatório Necessidades Educativas Especiais na Europa da Agência Europeia para o Desenvolvimento em Necessidades Educativas Especiais (AEDNEE), de 2003 sugere que o acesso "inadequado ou limitado às TIC" (AEDNEE, 2003) pode promover desigualdades entre alunos, alertando para a necessidade das políticas, dos projetos e programas com TIC contemplarem a formação dos professores, a atribuição de hardware e de software adequado e adaptado, a promoção da investigação, da inovação e da partilha de informação, e ainda, a sensibilização da sociedade sobre as vantagens das TIC para os alunos com NEE.

Segundo este relatório, é essencial refletir sobre o uso das TIC como instrumento de aprendizagem (em contextos variados) para a promoção da "verdadeira inclusão das TIC no currículo dos alunos com necessidades educativas especiais”. 


\subsection{TIC e T21}

As TIC ganham importância nos processos de aprendizagem, enquanto ferramentas de desenvolvimento em indivíduos portadores de T21.

Segundo Feng, Lazar, Kumin e Ozok (2008) este desenvolvimento ocorre em meios em que as TIC são uma presença constante e envolvente, embora existam poucos estudos descritivos sobre como é que os portadores de T21 efetivamente usam as TIC.

Black e Wood (2003) e Wood (2004) descrevem o conjunto de características das TIC que as tornam particularmente ajustadas ao estilo e aumentam a auto estima ao promover a autonomia, oferecem feedback imediato, permitem a aprendizagem no sentido do sucesso, facilitam a prática repetida, permitem ainda a aprendizagem individualizada, oferecem áreas de trabalho organizadas e previsíveis, podem ser adequadas às necessidades específicas de cada utilizador.

A investigação levada a cabo por Kirijian, Myers e Charland (2007), revela que, por exemplo, o tipo de letra Comics Sans Serif5 ou o efeito Drop Caps6 não são escolhas dos utilizadores portadores de T21. Verifica-se, igualmente, que estes utilizadores preferem botões grandes e bem identificados e que imagens de pessoas, especialmente de pessoas portadoras de T21, são preferidas a quaisquer outras imagens.

O estudo realizado por Feng, Lazar, Kumine e Ozok. (2008) revela que os utilizadores de TIC com T21 estão familiarizados com o teclado, o rato, o monitor e a impressora, verificando-se que, dão preferência à utilização do rato face ao ecrã tátil, apesar dos movimentos do cursor no monitor serem abstratos e requererem competências de motricidade fina, embora estas competências estejam associadas a características pouco desenvolvidas nestes indivíduos (Fodler et al, 2006).

Ainda de acordo com Feng, Lazar, Kumin e Ozok (2008) estes indivíduos não revelam dificuldades no uso de rato, o que demonstra a importância das capacidades motoras face às cognitivas e espaciais no sucesso na utilização de TIC por portadores de T21. Também Almeida (2006) refere a capacidade destes sujeitos para utilizar o rato e o teclado. Ainda segundo Almeida (2006, p. 12), estes utilizadores apresentaram níveis elevados de motivação o que poderá estar relacionado com o baixo nível de "experiência computacional e literacia tecnológica que estas crianças apresentavam no início do estudo, (...) e com (...) a «novidade» da experiência”. 


\subsection{As TIC aplicadas às NEE}

Cnoti e a Imagina é uma empresa de inovação centrada na área da aprendizagem, envolvida pelas tecnologias de Softwares e hardwares inclusivos, direcionando a sua pesquisa e desenvolvimento em soluções integradas para a educação. Tem por finalidade ajudar crianças, jovens e educadores a pensar, aprender e interagir com as tecnologias emergentes, valorizando as suas inteligências múltiplas, promovendo a participação e a inclusão (CNOTINFOR, 2008).

O Soundbeam é uma ferramenta de intervenção, um hardware e software, direcionado para crianças, jovens e adultos com necessidades educativas especiais, como elo de ligação entre a música, as emoções, o corpo em paralelismo pedagógico com o ensino e a educação (Wiliams, 2016).

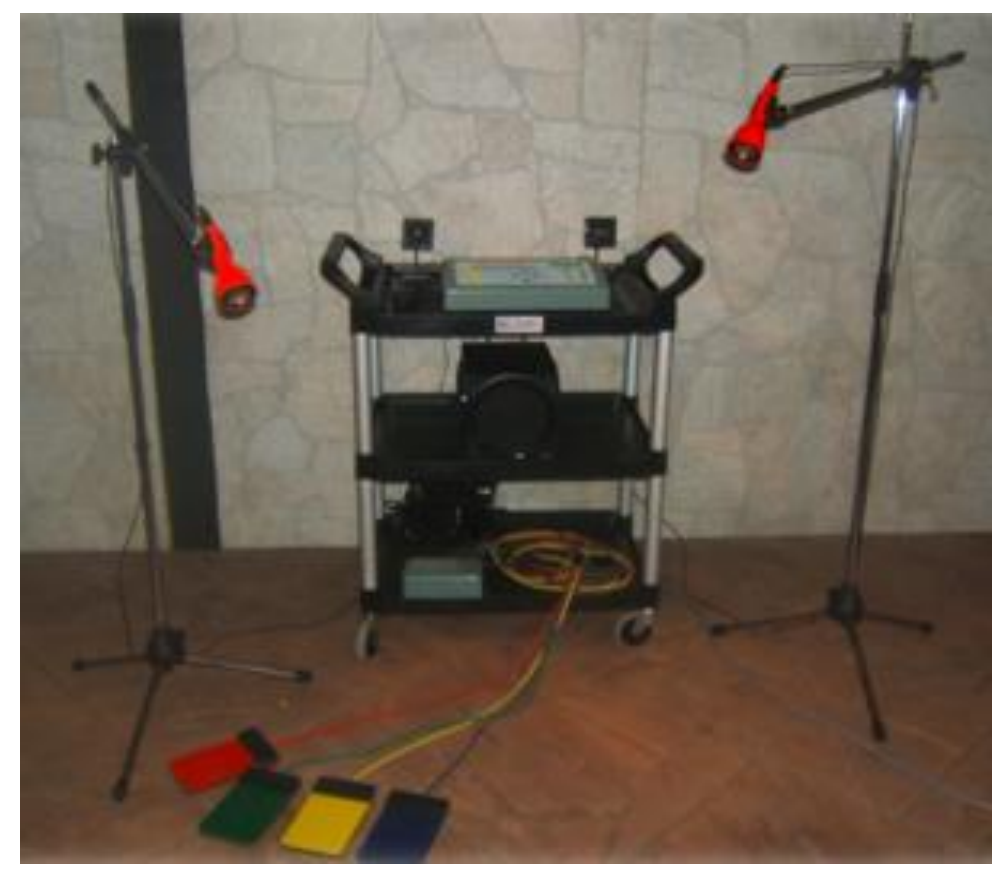

Figura 1 - Soundbeam 5.

O Soundbeam como meio tecnológico de conversão de movimentos físicos em som, foi concebido para proporcionar às crianças com NEE, uma participação mais ativa e de forma mais completa. "No seu processo educacional há um reforço da motivação, da atenção, da autoexpressão espontânea, da comunicação emocional e interação social” (Lee, 2015, p.3). O SoundBeam 5 é constituído por 3 sensores de movimento e 4 pedais de contacto físico através das mãos ou dos pés. 


\section{CONCEITOS MUSICAIS DE RITMO}

Segundo Cordero (2014, p. 173) "O ritmo é a vida de toda vida, sem ritmo nada pode existir. Sem uma consciência rítmica nada pode realizar-se. Assim ele está presente em todas as atividades que o homem realiza". "O ritmo é um elemento que pode beneficiar a conceção psicomotora do ser humano. É um movimento ou ruído que se repete, no tempo, em intervalos regulares, com acentos fortes e fracos" (Heller, 2012, p. 16). "A atividade é um principio fundamental em educação musical pois desenvolve a capacidade de observação e atenção levando o individuo a envolver-se e a participar efetivamente" (Rocha, 2014, p. 81). A “motricidade, a coordenação psico-motora é importante para o desenvolvimento global do individuo e para a prática da execução musical, podendo ser trabalhada através de exercícios de expressão verbal e vocal, percussão corporal, técnica instrumental, movimento" (Wuytack, 2016, p. 56).

\section{METODOLOGIA}

\subsection{Problemática}

A exposição de um problema representa a fase mais sensível de um processo de pesquisa. Significa dizer de forma clara qual a complexidade com que nos conferimos e queremos resolver (Tuckman, 2005, p. 22). Pretende-se compreender e colmatar uma dificuldade de marcação do ritmo, recorrendo a algumas estratégias, como meio para atingir objetivos e resultados positivos, no processo de ensino aprendizagem. Tendo por base, todos os princípios analisados e a consciência da necessidade e importância de investigar e refletir sobre as práticas educativas, toda a intervenção pedagógica foi pautada pela procura em responder à seguinte questão:

\section{Q1: A utilização de uma estratégia de ensino com recurso ao SoundBeam produz efeitos nos resultados de aprendizagem na Educação Musical?}

Explorar uma indicação de resposta para o problema, instrumento musical SoundBeam, descrevendo-o de forma clara, na qual possa ser testável e possível de reformulação (Tuckman, 2005, p. 23) e neste sentido, dar resposta à nossa questão acima transcrita, formulamos as seguintes hipóteses: 
Ho: A utilização de uma estratégia de ensino com recurso ao SoundBeam não produz efeitos nos resultados de aprendizagem ao nível da Educação Musical.

H1: A utilização de uma estratégia de ensino com recurso ao SoundBeam produz efeitos nos resultados de aprendizagem ao nível da Educação Musical.

Como opção metodológica foi utilizado um processo hipotético-dedutivo ${ }^{4}$, com objetivo exploratório ${ }^{5}$ e procedimento de pesquisa pré-experimental aplicada ${ }^{6}$ de natureza qualitativa. Esta intervenção pedagógica foi apenas aplicada em contexto de sala de aula. A estratégia adotada para a técnica de recolha de dados, fez-se através de grelha de registo em formato de pré e pós-registo, constituído por um conjunto de exercícios rítmicos, com estratégias aplicadas e que será alvo de análise na fase posterior deste documento. Formular hipóteses, proceder a observações e comparar resultado.

\subsection{Participantes}

Uma aluna de 23 anos de idade, com Síndrome de Down/Trissomia 21 regular (SD/T21). O processo de ensino aprendizagem foi aplicado e desenvolvido numa Unidade de Apoio Especializado à Multideficiência.

\subsection{Instrumentos e Procedimentos}

Em função das orientações curriculares do Ministério da Educação, pelo que nos orientamos ao nível das competências harmónicas, rítmicas e melódicas, destacamos as rítmicas a desenvolver intensamente com a referida aluna. Neste contexto o que nos propomos a desenvolver é o princípio imitativo por forma a obter reações espontâneas, no sentido da realização de padrões rítmicos simples, realizados anteriormente e de forma estratégica. “A aprendizagem da língua materna começa pelo processo de imitação, nesse sentido a primeira etapa do processo de aprendizagem da música deverá ser a imitação" (Wuytack, 2016, p.5). Os resultados obtidos serão sempre qualificados de forma estreita com a reação do individuo em tempo ou por defeito do objeto rítmico apresentado. $\mathrm{O}$ plano de intervenção terá como meio de registo uma grelha com valores qualitativos e pré-definidos em função do objetivo que se pretende obter, os resultados.

\footnotetext{
4 «Parte de um problema e da elaboração de hipóteses, envolvendo a criatividade e a imaginação, submetidas a critérios lógicos e empíricos, deduzindo-se delas consequências e procurando-se refuta-las» (Borges, 2007, p. 37).

5 "Proporcionar maior familiaridade com o problema, com vista a torna-lo mais explícito ou a construir hipóteses" (Gerhardt \& Silveira, 2009, p. 35).

${ }^{6}$ Apontamentos da unidade curricular de Metodologia de Investigação (Morais, 2014).
} 


\subsection{Estrutura da pesquisa}

Este processo, foi realizado entre os meses de novembro de 2016 e janeiro de 2017, com duração de 4 seções de 20 minutos cada. As sessões foram dedicadas ao seu design e procedimento no que diz respeita à sua aplicação prática. Os dados foram obtidos por registo da experiência prática. Este processo de ensino aprendizagem, desenvolveuse em três fases, conforme mais adiante será apresentado:

1) Estratégia $-1^{a}$ fase - o professor faz o exercício 3 vezes e a aluna observa;

2) Estratégia $-2^{\mathrm{a}}$ fase $-\mathrm{A}$ aluna repete em simultâneo com o professor o mesmo exercício 3 vezes;

3) Estratégia $-3^{a}$ fase - a aluna executa o exercício individualmente, recorrendo à visão, audição, concentração e memorização;

Para cada estratégia existiu uma preparação dos dados, seguida de um pré-registo, operacionalização, explicação, implementação, realização e resolução do problema, seguindo-se uma análise, avaliação e discussão dos resultados.

\subsubsection{Pré-experiência prática:}

Tabela 1 - Plano de intervenção

Execução Rítmica individual por imitação: aplicação no pedal do SoundBeam

\begin{tabular}{|c|c|c|c|c|c|c|c|c|c|c|c|}
\hline \multirow[b]{2}{*}{ Padrão rítmico } & \multirow[b]{2}{*}{$\begin{array}{l}\stackrel{\circ}{0} \\
\dddot{\Xi}\end{array}$} & \multirow[b]{2}{*}{$\begin{array}{l}\stackrel{8}{0} \\
\stackrel{0}{2} \\
\stackrel{8}{8}\end{array}$} & \multicolumn{2}{|c|}{ Pulsação } & \multicolumn{2}{|c|}{$N^{o}$ de figuras } & \multicolumn{3}{|c|}{ Intensidade } & \multicolumn{2}{|c|}{ Valores e Atitudes } \\
\hline & & & נֶّ & 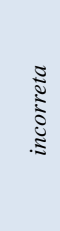 & ठัँ & 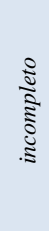 & 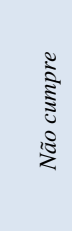 & 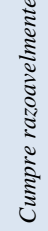 & 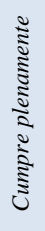 & 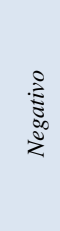 & 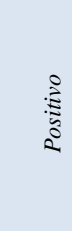 \\
\hline$\#$ d & $\mathrm{X}$ & & & $\mathrm{X}$ & $\mathrm{X}$ & & & & & & $\mathrm{X}$ \\
\hline$\| \cdot d \cdot d \cdot \mid$ & $\mathrm{X}$ & & & $\mathrm{X}$ & $\mathrm{X}$ & & & & & & $X$ \\
\hline$\|$ & $\mathrm{X}$ & & & $\mathrm{X}$ & $\mathrm{X}$ & & $\mathrm{X}$ & & & & $\mathrm{X}$ \\
\hline d. & $\mathrm{X}$ & & & $\mathrm{X}$ & $\mathrm{X}$ & & $\mathrm{X}$ & & & & $\mathrm{X}$ \\
\hline
\end{tabular}

Grelha de avaliação: padrões rítmicos

$1^{\circ}$ plano de intervenção: pressionar pedal do SoundBeam, na execução dos Padrões Rítmicos

- A aluna sentiu dificuldade na execução dos padrões rítmicos pedidos. 
Após um trabalho de campo sistemático e regular, recorrendo a diversas estratégias de ensino aprendizagem, tais como diferentes batimentos corporais (dedos, palmas, joelhos, pés), utilização de variados instrumentos musicais (clavas, bloco de dois sons, reco-reco, pandeireta, guitarra), na execução de padrões rítmicos, tanto a nível individual como em grupo, verificou-se que surtiu resultados positivos, como são apresentados na tabela abaixo:

\subsubsection{Pós-experiência prática:}

Tabela 2 - Plano de intervenção

\begin{tabular}{|c|c|c|c|c|c|c|c|c|c|c|c|}
\hline \multirow[b]{3}{*}{ Padrão rítmico } & \multicolumn{11}{|c|}{ Execução Rítmica individual por imitação: aplicação no pedal do SoundBeam } \\
\hline & \multirow[b]{2}{*}{$\begin{array}{l}\stackrel{8}{0} \\
\approx \\
\approx\end{array}$} & \multirow[b]{2}{*}{ 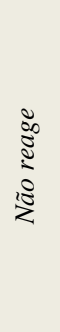 } & \multicolumn{2}{|c|}{ Pulsação } & \multicolumn{2}{|c|}{$N^{o}$ de figuras } & \multicolumn{3}{|c|}{ intensidade } & \multicolumn{2}{|c|}{ Valores e atitudes } \\
\hline & & & نَّ & $\begin{array}{l}\frac{\pi}{3} \\
\stackrel{\Xi}{0} \\
\stackrel{\Xi}{0}\end{array}$ & $\frac{8}{\stackrel{8}{\Xi}}$ & 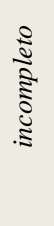 & 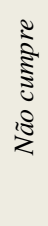 & 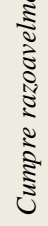 & 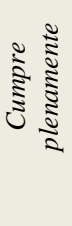 & 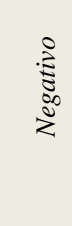 & $\begin{array}{l}\stackrel{0}{\vdots} \\
0 \\
0 \\
0\end{array}$ \\
\hline$\| \begin{array}{llll}\| & d\end{array}$ & $\mathrm{X}$ & & $\mathrm{X}$ & & $\mathrm{X}$ & & & & & & $\mathrm{X}$ \\
\hline$\| \cdot \cdot \cdot \cdot \cdot \mid$ & $\mathrm{X}$ & & $\mathrm{X}$ & & $\mathrm{X}$ & & & & & & $\mathrm{X}$ \\
\hline 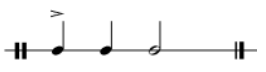 & $\mathrm{X}$ & & $\mathrm{X}$ & & $\mathrm{X}$ & & & & $\mathrm{X}$ & & $\mathrm{X}$ \\
\hline II & $\mathrm{X}$ & & $\mathrm{X}$ & & $\mathrm{X}$ & & & & $\mathrm{X}$ & & $\mathrm{X}$ \\
\hline
\end{tabular}

Grelha de avaliação: padrões rítmicos

$\underline{2^{\circ} \text { plano de intervenção: }}$ pressionar o pedal do SoundBeam, na execução dos Padrões Rítmicos.

- A aluna conseguiu executar plenamente os padrões rítmicos pedidos, mas apenas conseguiu repetir corretamente o exercício 3 vezes, em seguida, a aluna começou a demonstrar dificuldade, em função do seu problema de saúde, o Síndrome de Down.

\section{RESULTADOS}

No sentido da resposta em função da estratégia usada no formato pré e pós-registo dos exercícios rítmicos apresentados, com o objetivo na confirmação da obtenção e retenção de conhecimento por parte da aluna, verificou-se no pré-registo que a aluna teve dificuldades em alcançar o pretendido, mas após terem sido trabalhados os exercícios com diferentes estratégias direcionadas para o pretendido, verificou-se que a mesma conseguiu com sucesso atingir os resultados desejados. 


\section{DISCUSSÃO}

Após uma análise detalhada apresentada pela comunidade científica sobre o tópico em estudo, verifica-se uma atenção cuidada sobre o SD e as suas implicações no ser humano. A criança com SD, ao ter um cromossoma a mais em cada célula, leva a que o seu Sistema Nervoso Central (SNC) se oriente de forma diferente, na abordagem à informação recebida e ao seu processamento. Toda essa informação, tanto visual como sensorial é, ou deve ser proporcionada ao individuo, para que o mesmo a possa desenvolver, o mais possível, em todos os aspetos motores e cognitivos.

\section{CONCLUSÃO}

Conclui-se que, o seu SNC processa a informação recebida de forma mais lenta do que deveria, levando à apresentação dos resultados e à sua execução, após o pretendido. Tal facto comprova, que a música produz um efeito biológico e atua bioquimicamente sobre o nosso organismo, altera o ritmo cardíaco em função do andamento mais ou menos acelerado da música. Aproveitando a dedicação e o gosto pelo conhecimento que a aluna demonstra, deduzimos que parte do problema será ultrapassado, se não tiver que repetir consecutivamente o que lhe é pedido, mas sim através de um processo gradual de reconhecimento da própria, pelo alcançar do que lhe é pedido. As vantagens desta estratégia de ensino-aprendizagem, centram-se na particularidade da partilha e entreajuda dos pares, como meio de descontração, concentração e realização pessoal ao nível cognitivo e motor das suas capacidades e dificuldades. As limitações poderão refletir-se do constrangimento que possa surgir na partilha, por motivos da diferença. Nesse sentido recomenda-se em primeiro lugar que o grupo/turma se conheça minimamente. Recomenda-se ainda que, para trabalhos futuros, um cuidado pormenorizado na recolha de informação e análise de cada situação que se nos apresente. É fundamental estar atento ao maior número de detalhes, tanto verbais como expressivos, por parte de quem necessita e procura informação. A pesquisa científica tem de estar sempre presente no processo de ensino-aprendizagem. É de concluir que todo este processo passa por imensas experiências, tanto profissionais como humanas. A paciência, a dedicação, a entrega à causa leva à sabedoria e com isso à satisfação. O sucesso deve-se à persistência, ao acreditar que se conseguirá algo. 


\section{REFERÊNCIAS BIBLIOGRÁFICAS}

Anunciação, L. M. R. L., Costa, M. P. R., \& Denari, F. E., (2015). Teaching Practices For Students With Down Syndrome And Childhood Education: A Focus On Motor Development. Revista Brasileira De Educação Especial, 21(2), 229-244. doi:10.1590/S1413-65382115000200005

Ataíde, A. M. F. E. (2008). A Educação Emocional Em Crianças Com Sindrome De Down (Curso De Especialização Em Educação Especial Domínio Cognitivo Motor). Escola Superior De Educação De Fafe, Fafe.

Barroso, S. C. P. G. (2010). Intervenção Precoce Em Síndrome De Down (Estudo De Caso). Escola Superior De Educação De Fafe, Fafe.

Borges, R. M. R. (2007). Em Debate: Cientificidade E Educação Em Ciências. (2a ed). Porto Alegre: Edipucrs.

Cordero, O. H. G. (2014). A Música, O Rítmo E A Educação Física. Revista Científica Faema, 5(2), 173-186.

Feng, J., Lazar, J., Kumin, L., \& Ozok, A. (2008). Computer usage by young individuals with down syndrome: An exploratory study. Proceedings of the 10th international ACM SIGACCESS conference on Computers and accessibility (Assets '08) (pp. 35-42). New York, NY, USA: ACM.

Gerhardt, T. E., \& Silveira, D. T. (2009). Métodos De Pesquisa. UFRGS, Brasil.

Heller, A. A. (2012). Ritmo, Motricidade, Expressão: O Tempo Vivido Na Música. Universidade Federal De Santa Catarina, Brasil.

Lee, L. (2015). Investigating the Impact Of Music Activities Incorporating Soundbeam Technology On Children With Multiple Disabilities. Journal of The European Teacher Education Network, 10, 1-12.

Morais, C. M. M. (2014). Metodologia Da Investigação Em Educação. Instituto Politécnico De Bragança.

Rocha, J. A. S. (2014). Prática de Ensino Supervisionada em Ensino de Educação Musical no Ensino Básico. Instituto Politécnico De Bragança, Bragança.

Wiliams, E. (2016). Soundbeam. Disponível em: Www.Soundbeam.Co.Uk/ Wuytack, J. (2016). Pedagogia Musical. Porto: Assoc. Wuytack de Pedagogia Musical. 